\title{
DYSLIPIDAEMIA
}

\section{Promising new therapy for lysosomal acid lipase deficiency}

A phase III trial of enzyme-replacement therapy for lysosomal acid lipase deficiency has shown the effectiveness and safety of sebelipase alfa, a recombinant lysosomal acid lipase. Lysosomal acid lipase deficiency causes pathological effects mainly in the liver as a result of lysosomal accumulation of neutral lipids in the hepatocytes. Disease progression leads to cirrhosis and severe dyslipidaemia. This deficiency, which is often under-recognized or misdiagnosed, has limited treatments.

Barbara Burton and colleagues evaluated the effectiveness and safety of sebelipase alfa therapy in a multicentre, randomized trial that included 66 children and adults with lysosomal acid lipase deficiency. A 20-week double-blind, placebocontrolled period was followed by ongoing open-label treatment for all patients. At the end of the controlled period, normalization of the alanine aminotransferase level (the primary end point) was significantly higher in the sebelipase alfa group than in the placebo group ( $31 \%$ vs $7 \%$; $P=0.03$ ).

Sebelipase alfa therapy was also associated with improvements in six secondary efficacy end points, including reductions in the hepatic fat content and liver volume, and improvement in the plasma lipid levels. The rate of adverse events was similar in each group.

These results suggest that sebelipase alfa is an effective enzyme-replacement therapy for lysosomal acid lipase deficiency. Further studies in a larger number of patients will be needed to assess the effects of sebelipase alfa on the long-term progression of liver disease, cardiovascular events, and mortality in patients with this deficiency.

Irene Fernández Ruiz

Original article Burton, B. K. et al. A phase 3 trial of sebelipase alfa in lysosomal acid lipase deficiency. N. Engl. J. Med. 373, 1010-1020 (2015) 